\title{
Caracterización Psiquiátrica y Social del Intento Suicida Atendido en una Clínica Infantil, 2003-2005
}

\author{
Psychiatric and social characterisation of suicide attempts treated at \\ a children's clinic, 2003-2005
}

Isabel Pérez-Olmos, Esther Rodríguez-Sandoval, Mónica M. Dussán-Buitrago y Juan P. Ayala-Aguilera

Facultad de Medicina, Universidad del Rosario, Bogotá, Colombia. iperez@urosario.edu.co, erodrisan63@yahoo.com.ar, Monimad@msn.com, Juan.ayala10@urosario.edu.co.

Recibido 22 Septiembre 2006/Enviado para Modificación 11 Abril 2007/Aceptado 14 Mayo 2007

\section{RESUMEN}

Objetivos Caracterizar psiquiátrica y socialmente el intento suicida atendido en una clínica infantil de Bogotá, entre 2003 y 2005.

Métodos Serie retrospectiva de 96 Casos de 11 a 18 años de edad. Se realizó análisis estadístico univariado y bivariado.

Resultados: Las mujeres constituyeron $81,4 \%$. La edad promedio fue 15,3 años y 70,9 \% tenía máximo 16 años. El 63,1 \% de familias tenían bajos ingresos. La intoxicación fue el método suicida del 96,9\%. El $38 \%$ tuvo seguimiento ambulatorio máximo por 2 meses. Sólo 13,2 \% requirió medicina antidepresiva o moduladora del afecto. El 87,9 \% tenía estresantes familiares o escolares. El 83,5 \% tenía problemas con los padres, y en $72,3 \%$, los padres tenían relación conflictiva. Eran primogénitos $46,5 \%$. Se encontraron 40 situaciones de maltrato. El 27,2\% tuvo intento suicida previo. Los trastornos adaptativos, la disfunción familiar y los trastornos del afecto fueron los principales diagnósticos psiquiátricos. El 37,6\% tenía problemas escolares, pérdida escolar o conflictos con los maestros. El sexo masculino se asoció con agresividad, consumo de sustancias, fracasos escolares o sentimentales y el femenino con ansiedad predominante y maltrato físico.

Discusión Se resalta la disminución de la edad, el exceso de representación femenina, la frecuencia alta de conflictos familiares o escolares y la asociación del sexo con perfiles específicos socioculturales, conductuales y emocionales. EI conflicto sociofamiliar y escolar es frecuente en el intento suicida juvenil. Es necesario hacer visible la problemática suicida infanto-juvenil para su prevención.

Palabras Clave: Intento de suicidio, relaciones familiares, trastornos de adaptación, adolescente, niño, Colombia (fuente: DeCS, BIREME). 


\section{ABSTRACT}

Objective Psychiatrically and socially characterising suicide attempts treated at a children's clinic in Bogotá, 2003-2005.

Methods A retrospective series of 96 cases aged 11-18, using univariate and bivariate statistical analysis.

Results $81,4 \%$ of the cases were female. Mean age was 15,3 years and $70,9 \%$ were aged 16 or younger. Low family income was found in $63,1 \%$. Poisoning was the suicide method in $96,9 \%$ cases. Ambulatory care for two months or less was continued in $38 \%$ cases. Only 13,2 \% required anti-depressive or mood-modulator medicine. $87,9 \%$ suffered family or scholastic stress. $83,5 \%$ had a problematic relationship with parents and in $72,3 \%$ of cases the parents had a conflictive relationship. 46,5\% were first-born children. Forty abuse situations were found. 27,2 $\%$ had attempted suicide previously. Adjustment disorders, family dysfunction and mood disorders were the main psychiatric diagnostics. 37,6 \% had scholastic problems, suffered academic failure or had conflict with their teachers. Males were associated with aggressiveness, substance use, academic failure or romantic loss and females were associated with predominant anxiety and physical abuse.

Discussion Being young, the overrepresentation of females and suffering scholastic and family conflict were noteworthy. Gender association with socio-cultural, behavioural and emotional profile is also worth noting. Socio-family and scholastic conflict is frequent in youth people's suicide attempts. The child-youth suicide problem must be made socially visible to prevent it.

Key Words: Suicide attempt, family relationships, adjustment disorders, adolescent, child, Colombia (source: MeSH, NLM).

$\mathrm{E}$ I suicidio es un problema de salud pública, la Organización Mundial de la Salud (OMS) lo señaló como 13ava causa de muerte, con 14,5 casos por 100.000 habitantes (1). El suicidio es 1 de las 3 primeras causas de muerte en el mundo entre los 15 y 34 años de edad (2). Aunque Estados Unidos (EU) tuvo altas tasas de suicidio juvenil, éstas han disminuido desde los 90`s; el tratamiento de la depresión juvenil y otros programas preventivos parecen haber contribuido (3-6). En España y Latinoamérica el fenómeno está en aumento $(2,5,7,8)$. En los últimos años, en Bogotá y Colombia, el grupo de 16 a 30 años de edad ha ocupado el primer lugar en frecuencia de suicidios (9). Los años de vida productiva perdidos por suicidio en 2003 fueron 64 343. Los métodos suicidas preferidos fueron la intoxicación (31 \%), arma de fuego (29 $\%)$ y ahorcamiento (20 \%) (10-11). En Colombia en 2003 murieron por suicidio 160 menores de edad, 86 \% mujeres; a su vez, el $25 \%$ de las 64 mujeres que se suicidaron en Bogotá en el mismo año eran menores de edad, 50 \% tenía 16 a 17 años. El $75 \%$ tuvo fácil acceso al método empleado (12). 
De los factores de riesgo (FR) para suicidio juvenil el más descrito es el intento suicida previo (5,6,11,13-15). También están la depresión mayor, el trastorno afectivo bipolar, la esquizofrenia, la personalidad limítrofe o sociopática $(5,6,10,13,15-21)$. El $90 \%$ de los suicidas adultos tiene al menos un trastorno psiquiátrico; en los jóvenes, sólo el 60 \% (4). El alcohol y sustancias de abuso son predictores de suicidio en personas con riesgo $(2,4-6,10,13)$. Cerca del 50 $\%$ de los suicidas están intoxicados al morir y 18 \% de los alcohólicos muere por suicidio (15). El $11 \%$ de los suicidas tiene un familiar de primer grado con muerte por suicidio (15). La biología señala bajas concentraciones del metabolito de la serotonina, ácido 5- hidroxindolacético, en suicidas comparados con controles. Estudios postmortem de suicidas y accidentados mostraron menor cantidad de receptores de serotonina en el tallo cerebral de los suicidas $(4,10,13,15)$. El fácil acceso a las armas de fuego representa un mayor riesgo para actos suicidas $(2,4,5,12,15-17)$. Las mujeres cometen el doble de intentos suicidas que los hombres; sin embargo, la muerte por suicidio ocurre 3:1 en los varones $(11,13)$. Ser víctima o generador de violencia es un FR para suicidio; por ejemplo, violencia intrafamiliar o participar en grupos violentos organizados $(5,17,19)$. Hay mayor ocurrencia de suicidio en los extremos del espectro socioeconómico. También se describe el efecto Werther o imitación de la conducta suicida juvenil difundida en medios masivos de comunicación (4$6,17,22,23)$.

Los FR psicológicos incluyen conflictos, pérdidas y cambios en las relaciones interpersonales y la desesperanza aprendida $(14,17)$. También, familias disfuncionales, maltrato o abuso sexual infantil, el sentimiento de soledad y conductas autodestructivas (6,8,24-28). En 2005, la autopsia psicológica de jóvenes suicidas de Utah, zona con las mayores tasas de suicidio de EU, señaló al estigma de la enfermedad mental como un factor limitante para su tratamiento (29). Entre los factores protectores (FP) del suicidio están las buenas relaciones significativas e interpersonales $(4,17)$. Aquellos con cohesión familiar, alto grado de involucramiento mutuo, intereses compartidos y apoyo emocional, eran 3,5 a 5,5 veces menos propensos al suicidio (4).

La historia clínica completa es el pilar diagnóstico, debe registrar intentos suicidas previos, gravedad de los mismos y la evaluación del riesgo suicida $(15,19,30,31)$. El sub-registro es un problema en el estudio del suicidio; en EU por cada suicidio conocido se calculan 4 no registrados. Hay intentos suicidas clasificados como intoxicaciones o accidentes $(5,13,14)$. El aumento del suicidio juvenil motivó a los autores a realizar esta caracterización psiquiátrica y social 
de los jóvenes atendidos en una Clínica Infantil de Bogotá por intento de suicidio, en búsqueda de medios para prevención.

\section{MATERIALES Y MÉTODOS}

Diseño. Serie de 96 casos, edad entre 11 y 18 años, atendidos por intento suicida en la Clínica Infantil de Colsubsidio del $1^{\circ}$ de Enero de 2003 al 31 de diciembre de 2005. La información de variables sociodemográficas, familiares y clínicas se obtuvo de las historias clínicas. Fueron atendidos en Psiquiatría 86 casos.

Análisis Estadístico. Sehizo análisis uni y bivariado, con SPSS 13.0, y EpiInfo 6.0, a base de datos hecha en Excel (Microsoft Office). Se usó un nivel de confianza de $95 \%$ y el Test Exacto de Fisher si era indicado.

Consideraciones Éticas. El estudio fue aprobado por el Comité de Bioética de la Clínica. En los datos se protegió la identidad de los jóvenes.

\section{RESULTADOS}

Características sociodemográficas y familiares. La relación mujer hombre fue 4:1, siendo mujeres el 81,4 \% ( $n=70)$. El promedio de edad fue 15,3 años (Desviación estándar 1,78), rango de 11 a 18 años. La edad más frecuente fue 16 años ( $25 \%, \mathrm{n}=24)$, los pacientes de 16 años o menos fueron el 71,9 \% correspondiente a 69 casos, el grupo de 14 años o menos fue de $26 \%(n=25)$ con más casos que las otras edades (Figura 1). No hubo asociación entre edad y sexo. Las familias de los 96 casos tenían al menos un progenitor afiliado al régimen contributivo en salud. En 84 historias con información, el 63,1 \% (n=53) de las familias eran de bajos ingresos y el 32,1 \% ( $n=27)$ de ingresos medios.

Figura 1. Distribución de edad por grupos. Intentos de suicidio de niños y adolescentes. Clínica Infantil de Colsubsidio. Bogotá 2003-2005.

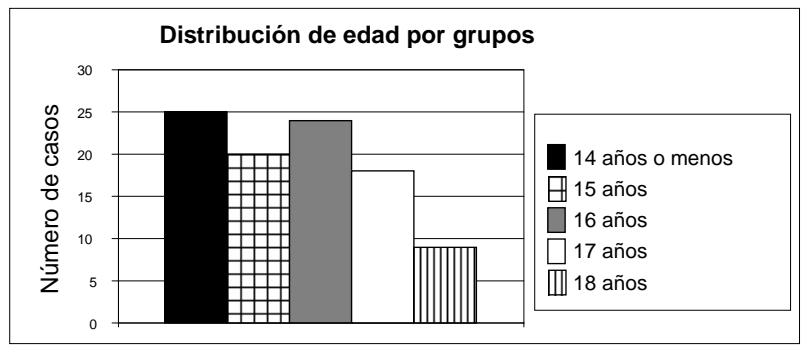


Características escolares. La escolaridad estuvo entre $5^{\circ}$ de primaria y $2^{\circ}$ semestre universitario. Ocurrió pérdida escolar en $24,7 \%$ ( $n=21)$ y 53,3 \% de los 15 casos $(\mathrm{n}=8)$ con esta situación se dieron en el mismo año del intento y el resto en el año anterior. Tenían problemas con maestros $32 \%(n=24)$ y problemas escolares al momento del intento suicida 37,6 \% (n=32).

Características familiares. El 43,2 \% (n=38) de jóvenes vivía con su familia nuclear, el 5,7 \% ( $n=5)$ con la familia extensa y el 51,1 \% (n=45) vivía sólo con uno de los padres. En cuanto al orden cronológico de los hijos en la familia, el $18,8 \%(n=18)$ eran hijos únicos, el 46,5 \% $(n=33)$ primogénitos y el 33,8 \% ( $\mathrm{n}=24)$ segundos hijos. El 83,5 \% (n=76) de los jóvenes tenía problemas con los padres, el 72,3 \% (n=60) de los padres tenía conflictos en su relación y el $58,8 \%(n=47)$ refería los problemas familiares como causa de estrés. El 60,9 $\%(n=56)$ de los padres estaban separados. El $54 \%(n=34)$ de casos con información $(\mathrm{n}=81)$, referían rivalidad entre hermanos. Había patología psiquiátrica familiar en 8,5 \% $(\mathrm{n}=6)$ y en 7,1 \% $(\mathrm{n}=5)$ suicidio familiar.

Factores detonantes. En 92 historias con información, el 83 \% (n=77) tenía al menos un evento detonante para el suicidio, conflictos familiares en $56 \%$ $(\mathrm{n}=54)$, conflictos sentimentales en $22,1 \%(\mathrm{n}=17)$ y problemas escolares en $14,3 \%(\mathrm{n}=11)$. De otro lado, los padres conocían del evento detonante en 52,6 $\%(n=41)$ de los 78 casos con esa información, el 78 \% (n=32) coincidían con los jóvenes en la problemática familiar como causa.

Factores estresantes. Se encontró que 87,9 \% (n=80) de las 91 historias con información, incluía al menos un evento estresante en la vida de los jóvenes. Los estresantes provenían del ambiente familiar, las relaciones sentimentales, los pares y el colegio, cada joven tuvo mínimo dos eventos estresantes (Tabla 1). Hubo 3 casos de pares suicidas, 2 de ellos murieron; los suicidios de los pares ocurrieron el mismo año del intento suicida del caso relacionado. Todos los casos de maltrato tenían al agresor en la familia. Hubo 40 situaciones de maltrato físico, psicológico o sexual, algunos jóvenes sufrieron más de un tipo de maltrato.

Hallazgos de la Evaluación Psiquiátrica. Todos los casos fueron atendidos en el Servicio de Urgencias de la institución. El Servicio de Psiquiatría valoró al 89,6 \% de los pacientes ( $\mathrm{n}=86$ ). El diagnóstico psiquiátrico más frecuente fue trastorno Adaptativo, seguido de Disfunción Familiar, Depresión u otros Trastornos del Afecto. Hubo más de un diagnóstico por caso (Tabla 2). La agresividad en 43,8 \% ( $n=35)$, tristeza 35,5\% ( $n=28)$, y baja autoestima 32,5 
$\%$ ( $\mathrm{n}=26$ ), fueron los estados mentales y emocionales predominantes, seguido por mal genio $28.8 \%(n=23)$ y ansiedad con $20 \%(n=16)$; hubo más de un estado mental o emocional en algunos pacientes.

Tabla 1. Eventos Estresantes. Intentos de suicidio de niños y adolescentes Clínica Infantil de Colsubsidio. Bogotá, 2003-2005

\begin{tabular}{lcc}
\hline \multicolumn{1}{c}{ Tipo de Evento estresante } & $\begin{array}{c}\text { número de } \\
\text { casos }\end{array}$ & $\%$ \\
\hline Familiares & 102 & 123 \\
\hline Conflictos familiares & 47 & 58,8 \\
\hline Maltrato físico & 18 & 20 \\
\hline Maltrato psicológico & 15 & 16,6 \\
\hline Duelo por muerte de Familiar & 11 & 13,8 \\
\hline Abuso sexual & 7 & 8,8 \\
\hline Económicos & 4 & 5 \\
\hline Relaciones & 46 & 53,8 \\
\hline Problema sentimental & 33 & 37,5 \\
\hline Embarazo & 4 & 5 \\
\hline Rechazo del grupo de pares & 6 & 7,5 \\
\hline Suicidio de un par & 3 & 3,8 \\
\hline Problemas Escolares & 23 & 28,8 \\
\hline Total eventos estresantes & 171 & 205,6 \\
\hline Sin estresante informado & 11 & 12,1 \\
\hline
\end{tabular}

Tabla 2. Diagnósticos en Psiquiatría. Intentos de suicidio de niños y adolescentes. Clínica Infantil de Colsubsidio. Bogotá 2003-2005

\begin{tabular}{lcc}
\hline Diagnóstico & Número de casos & \% con el diagnóstico \\
\hline Trastorno adaptativo & 42 & 50 \\
Disfunción familiar & 32 & 38 \\
Depresión u otro trastorno del afecto & 15 & 18 \\
Trastorno del control de Impulsos & 7 & 8 \\
Retardo mental & 2 & 2 \\
Pobreza & 1 & 1 \\
\hline
\end{tabular}

Perfil suicida: La intoxicación fue el método suicida del 96,9 \% (n=93) y 17,8 \% (n=16) ingirieron alcohol adicionalmente. El 79,8 \% (n=75) no avisó del intento y el 85,6\% ( $\mathrm{n}=77)$ no tenía un plan suicida; sin embargo, 27,2 \% (n=25) había intentado suicidarse previamente. La información sobre intento suicida previo faltó en el 4,2 \% $(n=4)$ de las historias clínicas. Los intentos previos iban entre 1 (n=19) y 4 intentos (n=1). El 64,7 \% de 17 casos con información $(\mathrm{n}=11)$ habían intentado suicidarse en el último año, y de éstos, 9 en los últimos 6 meses.

No tuvieron seguimiento psiquiátrico el 46,7 \% $(\mathrm{n}=43)$ de los casos, el 38 $\%(\mathrm{n}=35)$ lo tuvo máximo por 2 meses y 15,2 \% $(\mathrm{n}=14)$ por 3 meses o más, con un año de seguimiento en 1 caso (Figura 2). El 42,6\% (n=12) tuvo 1 o 2 consultas de control y el 11,7 \% ( $\mathrm{n}=11$ ) tres controles o más, con un máximo de 7 controles en 1 paciente. Se formuló medicación ambulatoria al 13,2 \% (n=9), 
antidepresivos o moduladores del afecto como fluoxetina, trazodone o carbamazepina; 2 pacientes suspendieron la medicación al no creerla necesaria. Como hallazgos adicionales dos casos repitieron el intento de suicidio durante esta investigación, uno a los 8 meses del primer intento; el segundo, lo informó en un control de psiquiatría. Dos casos tuvieron problemas con la ley por hurtos menores.

Consumo de alcohol y sustancias. El 12,8 \% ( $\mathrm{n}=10)$ tenía consumo frecuente de alcohol, el 2,6 \% $(\mathrm{n}=2)$ abusaba del alcohol y no había información en 18 casos. Había consumo de sustancias psicoactivas en 14,6 \% ( $\mathrm{n=14}$ ) de 84 historias con esta información; el 4,2 \% ( $n=4)$ consumía cigarrillo, 4,2 \% (n=4) marihuana, el 4,2 \% ( $n=4)$ cocaína, el $1 \%$ (n=1) combinaba éxtasis con cocaína y el $1 \%(n=1)$ usaba morfina.

Figura 2. Tiempo de seguimiento ambulatorio. Intentos de suicidio de niños y adolescentes. Clínica Infantil de Colsubsidio. Bogotá 2003-2005

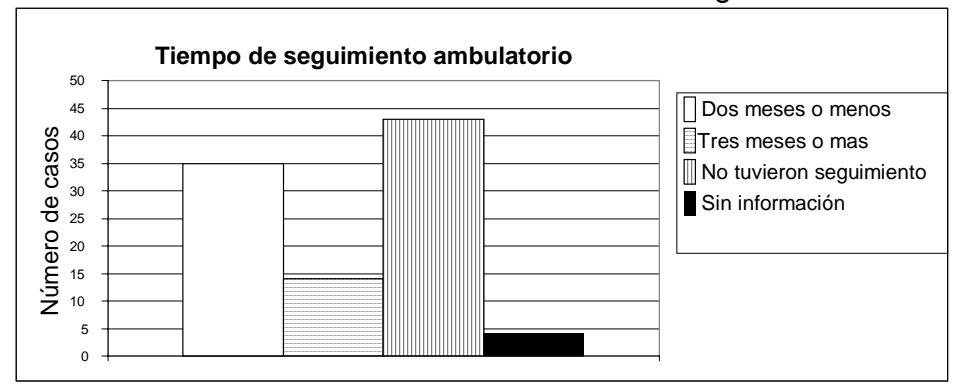

Análisis Bivariado. El intento suicida de los hombres se asoció con agresividad como emoción predominante, $\mathrm{X}^{2}$ (Yates) 3,9 P = 0,048, Riesgo relativo (RR) 1,8 (IC 1,2, 2,9); con el consumo habitual de sustancias, Test exacto de Fisher: $\mathrm{P}=$ 0,02; RR 3,2 (IC 1,3, 7,9), problemas en la relación sentimental, Test de Fisher: $\mathrm{P}=0,012$; RR 3,3 (IC 1,5, 7,0) y pérdida escolar: Test de Fisher: $\mathrm{P}=$ 0,05; RR 2,2 (IC 1,04, 4,5). El intento suicida de las mujeres se asoció con ansiedad como emoción predominante, Test exacto de Fisher: $\mathrm{P}=0,018$. A su vez, el maltrato físico estuvo asociado con el intento suicida de las mujeres, Test exacto de Fisher: $\mathrm{p}=0,021$.

\section{DISCUSIÓN}

Por cada hombre, 4 mujeres intentaron suicidarse, esta distribución excede en representación femenina la señalada en la literatura $(11,13)$. El exceso femenino podría relacionarse con la depresión, el maltrato, el abuso sexual infantil y la 
violencia intrafamiliar, cuyas víctimas predominantes son las mujeres; fenómeno de alta prevalencia en nuestro país, además de otras formas de inequidad de género de la sociedad colombiana (32).

Las edades cercanas a 15 años concuerdan con la menor edad del suicida señalada en la literatura (1-5). Este hecho se ha explicado por alteración del balance bio-psico-social en la adolescencia; una pubertad más temprana y una dependencia social más duradera, causa estrés, tensiones y problemas en familias, colegios y sociedad $(5,17,19,33,34)$. De otro lado, el nivel escolar de los casos fue concordante con la edad. Las pérdidas de años escolares, y los conflictos con los maestros fueron frecuentes, la literatura los señala como desencadenantes de conducta suicida infantil (34). Los primogénitos podrían estar más presionados en el desbalance bio-psico-social descrito, sin embargo no se encontró mención de ello en la literatura revisada. Los detonantes familiares hallados concuerdan con los de suicidios femeninos de menores del Instituto Nacional de Medicina Legal y Ciencias Forenses en 2003 (12).

Los diagnósticos psiquiátricos fueron similares en estudios españoles, especialmente la depresión y trastornos del afecto (34), así como la duración del seguimiento ambulatorio (34). Algunos casos mostraron la tendencia a repetir el intento suicida como si fuese FR para nuevos intentos $(5,12-17,34)$. Los dos jóvenes que murieron, ya lo habían intentado antes. La conducta suicida no es explicable por una causa única, surge de múltiples factores de naturaleza biológica, psicológica y social. En la serie estudiada, el efecto psicológico de los detonantes parece adicionarse al estrés acumulado en la vida de los jóvenes, concordando así con los factores proximales y distales del suicidio de Moscicki $(15-17,34)$.

Algunos FR pueden ser objeto de programas de prevención; se encontró que los hombres tenían agresividad como emoción predominante, consumo habitual de alcohol y otras sustancias, y pérdidas sentimentales o escolares; la agresividad se ha encontrado en hombres de otros estudios; a su vez, los intentos de las niñas se asociaron con maltrato físico y ansiedad, esto es modificable con programas de educación y prevención en salud (4,12,15,17,32,35-38). Identificar factores modificables es el paso previo a la implementación de programas de prevención que apoyen la función protectora de la infancia en la familia, la escuela y la sociedad.

El estigma de los trastornos mentales debe ser superado para reducir la tasa de suicidio asociada (29). Las personas que necesitan servicios de salud 
mental deben ser vistas como personas que requieren cuidados básicos en salud $(29,30)$.

Limitaciones: Los diseños descriptivos sólo muestran tendencias, las hipótesis de causalidad necesitan ser probadas con otro tipo de estudios

Agradecimientos. A las Dras. Amparo Buendía, Jefe de Educación Médica, Olga Baquero, Pediatra del Servicio de Urgencias, Olga Higuera, Jefe del Servicio de Rehabilitación, y la Jefe de Atención al Cliente, María Fernanda Santacruz de la Clínica Infantil de Colsubsidio por su valioso apoyo a la realización de este proyecto.

\section{REFERENCIAS}

1. Krug EG, Dahlberg L, Mercy J, Zwi A, Lozano R. World report on violence and health. World Health Organization. Geneva; 2002.

2. World Health Organization. Burden of Mental and Behavioral Disorders. The World Health Report; 2001, p 37-38.

3. Saffer D, Pfeffer C, and The Work Group on Quality Issues. Practice Parameter for the Assessment and Treatment of Children and Adolescents with Suicidal Behavior. American Academy of Child and Adolescent Psychiatry. J Am Acad Child Adolesc Psychiatry. 2001 Jul;40(7 Suppl):24S-51S.

4. Gould M, Greenberg T, Velting DM, Shaffer D. Youth suicide risk and preventive interventions: a review of the past 10 years. J Am Acad Child Adolesc Psychiatry. 2003 Apr;42(4):386-405.

5. Duarte C, Bordin I, Hoven C. Conductas suicidas en jóvenes brasileños; análisis exploratorio de prevalencia y factores de riesgo. Psiquiatría y salud integral. 2002;2(4):32-37

6. Pelkonen M, Marttunen M. Child and adolescent suicide. Epidemiology, risk factors, and approaches to prevention. Pediatr Drugs. 2003;5(4):243-65

7.Venceslá JF, Soriana JA. Conducta autolítica y parasuicida. Características socio demográficas en población infanto juvenil de ámbito rural. Rev. Asoc. Esp. Neuropsiq. 2002; 21(84):49-64

8. Larraguibel M, González P, Martínez V, Valenzuela R. Factores de riego de la conducta suicida en niños y adolescentes. Rev.Chil. Pediatr. 2000; 71(3):1-13.

9. González J, Merchan C, MartheA, Lopez C, RicaurteA, RestrepoA, et al. Suicidios en Colombia 2001. En: Instituto Nacional de Medicina Legal y Ciencias Forenses. Forensis 2001. Bogotá: Imprenta Nacional; p.167-191.

10. Alejo H, González J, Hernández W. Conducta suicida según ciclo vital. En: Instituto Nacional de Medicina Legal y Ciencias Forenses. Forensis 2003. Bogotá: Imprenta Nacional; p.107-119 
11. Secretaria de Salud de Bogota. Boletín Epidemiológico Distrital. 2002; 7(6-9): 58.

12. Bohórquez M, Espinosa J, Lopez L, Pareja L, Sanchez A, Gonzalez J, et al. Suicidio y niñez. Factores relacionados con el suicidio en mujeres menores de 18 años en Bogotá durante el año 2003. En: Instituto Nacional de Medicina Legal y Ciencias Forenses. Forensis 2004. Bogotá: Imprenta Nacional; p. 185-226.

13. Gómez-Restrepo C, Rodríguez N, Bohórquez A, Diazgranados N, Ospina MB, Fernández C. Factores asociados al intento de suicido en la población colombiana. Rev Colomb Psiquiatr. 2002; 31(4): 271-86

14. Barón O. Adolescencia y suicidio. Psicología desde el Caribe. 2000; (6): 48-69

15. Maris RW. Suicide. Lancet. 2002 jul 27;360(9329):319-26.

16. Forero L, Cubillos D, Pérez-Olmos I. Caracterización del suicidio en Bogotá durante el año 2000 y exploración de sus factores asociados [Tesis de Grado]. Bogotá: Universidad del Rosario; 2001.

17. Campo G, Roa JA, Pérez A, Salazar O, Piragauta C, López L. Et al. Intento de suicidio en niños menores de 14 años atendidos en el hospital universitario del Valle, Cali. Colomb Med. 2003; 34(1):9-16

18. Tejedor MC, Pericay JM, Castillón JJ. Epidemiología del suicidio: factores individuales y sociales. Jano. 1988; 2(9):9-14.

19. Committee on adolescents. Suicide and suicide attempts in adolescents. Pediatrics. 2000 apr;105(4 pt 1):871-4.

20. Morales M, Jiménez I. El suicidio desde la perspectiva forense. Rev Colomb Psiquiatr. 1996; 25(1):29-37

21. Saffer D, Scott M, Wilcox H, Maslow C, Hicks R, Lucas CP, et. al. The Columbia suicide screen: validity and reliability of a screen for youth suicide an depression. J Am Acad Child Adolesc Psychiatry. 2004 jan;43(1):71-9.

22. Sánchez R, Guzmán Y, Cáceres H. Estudio de la imitación como factor de riesgo para ideación suicida en estudiantes universitarios adolescentes. Rev Colomb Psiquiatr. 2005;34(1): 12-25

23. Melhem N, Day N, Shear MK, Day R, Reynolds C and Brent D. Traumatic grief among adolescents exposed to a peer's suicide. Am J Psychiatry 2004; 161: 1411-16.

24. Brown J, Cohen P, Johnson J, Smailes EM. Childhood abuse and neglect: specificity of effects on adolescent and young adult depression and suicidality. J Am Acad Child Adolesc Psychiatry 1999 Dec; 38 (12): 1490-96.

25. Beautrais A, Joyces P, Mulder R. Risk factors for serious suicide attempts among youths aged 13 through 24 years. J Am Acad Child Adolesc Psychiatry 1996;35:1174-82.

26. Gould M, Shaffer D, Fisher P, Garfinkel R. Separation-divorce and child and adolescent completed suicide. J Am Acad Child Adolesc Psychiatry 1998; 37:155-62. 
27. McKeown RE, Garrison CZ, Cuffe SP, Waller JL, Jackson K, Addy C. Incidence and predictors of suicidal behaviors in a longitudinal sample of young adolescents. J Am Acad Child Adolesc Psychiatry 1998; 37: 612-9.

28. Groholt B, Ekeberg O, Wichstrom L, Haldorsen T. Suicidal and nonsuicidal adolescents; different factors contribute to self-esteem. Suicide Life Threat Behav. 2005Oct;35(5):525-35.

29. Moskos M, Olson L, Halbern S, Keller T, Gray D. Utah Youth Suicide Study: Psychological Autopsy. Suicide Life Threat Behav. 2005 Oct;35(5): 236-47.

30. Espinosa A, Anzures B. Suicidio, homicidio y drogadiccion en niños y adolescentes. Rev Med Hosp Gen (Mex). 1999; 62(3): 183-190.

31. Hirschfeld RM, Russell JM. Assessment and Treatment of Suicidal Patients. N Engl J Med. 1997 Sep 25; 337(13):910-5.

32. Pérez-Olmos I. Delito sexual, dictámenes sexológicos por delito sexual. Análisis de la Información forense Colombia 2001. En: Instituto Nacional de Medicina Legal y Ciencias Forenses. Forensis 2001. Bogotá: Imprenta Nacional; p. 128164.

33. Diekstra RFW. Depression and Suicidal Behaviors in adolescent: Sociocultural and time trends. In: Rutter M, editor. Psychosocial Disturbances in Young People. Challenges for Prevention. London: Cambridge University Press; 1997. p. 212-243.

34. Gastaminza X, Vacas R, Ros SM. La Conducta Suicida en la Infancia y en la Adolescencia. En: Ros SM(Ed.) La Conducta Suicida. Madrid: Arán ediciones S.A. 1998. P179-195.

35. Perez-Olmos I, Pinzon AM, Gonzalez-Reyes R, Sanchez-Molano J. Influence of violent TV upon children of a public school in Bogotá, Colombia. Rev. Salud Publica (Bogota) 2005 Mar; 7(1):70-88.

36. Philips MR, Li X, Zhang Y, Suicide Rates in China, 1995-1999. Lancet 2002; 359 (9):835-40.

37. Sánchez R, Orejarena S, Guzmán Y. Características de los suicidas en Bogotá: 19852000. Rev. Salud Pública (Bogotá) 2004; 6(3):217-23.

38. Brent DA, Baugher M, Bidge J, Chen T, Chiappetta L. Age and sex related risk factors for adolescent suicide. J Am Acad Child Adolesc Psychiatry 1999;38(12):1497-05. 